\title{
Ammonites Leonhardianus Karsten, 1858 de los depósitos del Cretácico Superior de Venezuela
}

Pedro Patarroyo ${ }^{1 *}$

Forma de citar: Patarroyo, P. (2021). Ammonites Leonhardianus Karsten, 1858 de los depósitos del Cretácico Superior de Venezuela. Boletín de Geología, 43(2), 15-28. https://doi.org/10.18273/revbol.v43n2-2021001

\section{Resumen}

Dentro de la colección de invertebrados fósiles del Museo de Historia Natural de Berlín (Alemania) se encuentra Ammonites Leonhardianus una nueva especie introducida por Karsten en 1858. Este taxón, Paralenticeras leonhardianus (Karsten, 1858) proviene de Barbacoas (municipio Morán, estado Lara - Venezuela) de una sucesión calcárea del Coniaciano que aparece al occidente de El Tocuyo. De la colección de P. leonhardianus (Karsten, 1858) se propone el lectotipo y tres paralectotipos, se describen, miden e ilustran 26 ejemplares de los que allí se hallan.

Palabras clave: Paralenticeras leonhardianus (Karsten, 1858); Cretácico Superior; Coniaciano; Venezuela.

\section{Ammonites Leonhardianus Karsten, 1858 from the Upper Cretaceous deposits in Venezuela}

\begin{abstract}
From an invertebrate fossils collection at the Museum of Natural History in Berlin (Germany) is possible to find Ammonites Leonhardianus that was introduced as new species by Karsten in 1858. This taxon, Paralenticeras leonhardianus (Karsten, 1858) was collected at the Barbacoas Village (Morán municipality, Lara state Venezuela) from a Coniacian calcareous succession to the west of El Tocuyo. To the collection of $P$. leonhardianus is proposed a lectotype and three paralectotypes. Here 26 specimens are described, measured and illustrated.
\end{abstract}

Keywords: Paralenticeras leonhardianus (Karsten, 1858); Upper Cretaceous; Coniacian; Venezuela. 


\section{Introducción}

El alemán Hermann Karsten (1817-1908) fue un profesional preparado en ciencias naturales con inclinación a la botánica. Al tener formación en ciencias naturales poseía conocimientos en geología y en paleontología, por lo que con el impulso y con el ejemplo de Alexander von Humboldt emprendió viaje hacia Suramérica en 1843.

Llega inicialmente a Venezuela en 1844 (CarrilloBriceño et al., 2016) en donde se encuentra con Agustín Codazzi (Aalto, 2015), quien allí adelantaba una cartografía geográfica detallada. Posteriormente Karsten pasa a Colombia alcanzando a llegar al norte de Ecuador. Con base en el reconocimiento de las rocas y en algunos casos con el contenido fósil, Karsten elaboró el primer mapa geológico de Venezuela, Colombia y el norte de Ecuador (Karsten, 1858, 1886, 1947; Aalto, 2015) incluyendo Panamá, que por aquel entonces hacia parte de Colombia. En 1947 aparece en Colombia una traducción del trabajo original de Karsten (1858) por lo que en esta contribución aparece la referencia Karsten (1947) pues se desconoce el nombre del traductor.

En sus investigaciones, Karsten estuvo en Venezuela inicialmente entre 1844 y 1847, posteriormente entre 1848 y 1852 (Carrillo-Briceño et al., 2016), en donde colectó fósiles en el norte y principalmente en cercanías a los Andes de Mérida (Karsten, 1849, 1850, 1858, 1886, 1947). Los fósiles recolectados por Karsten fueron llevados a Alemania, por lo que se encuentran almacenados y custodiados en el Museo de Historia Natural de Berlín (Museum für Naturkunde MNK) dentro de la colección Karsten. De los fósiles de Venezuela está Ammonites Leonhardianus (Karsten, 1858, pl. 2, fig. 5), especie hallada en Barbacoas (Figura 1), municipio Morán, estado Lara (Venezuela), que se ubica al occidente de El Tocuyo (cf. Renz, 1982, fig. 2; González et al., 2018). El poblado de Barbacoas se localiza en 949’33” N y 70³’12” W.

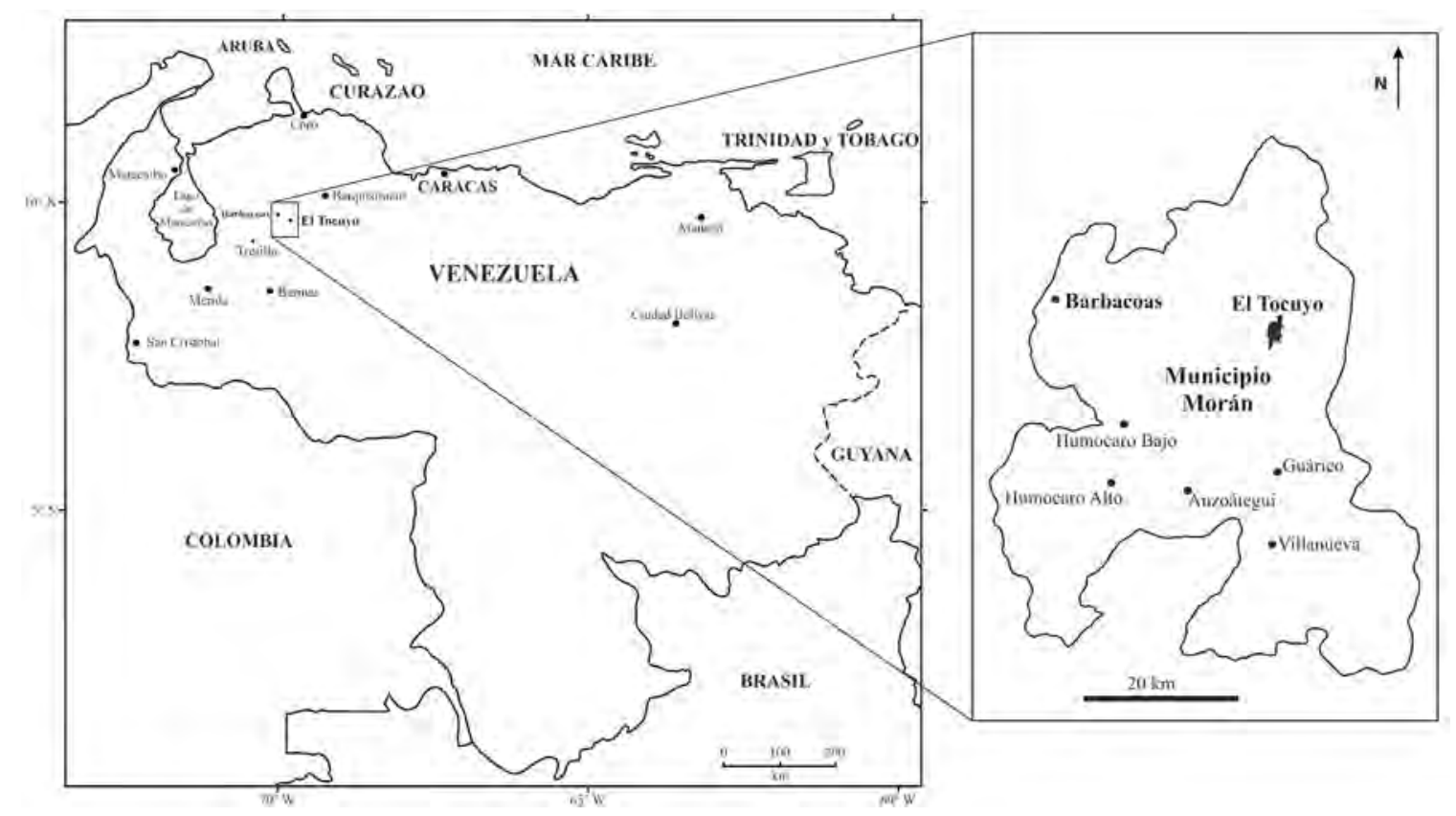

Figura 1. Mapa de localización de Venezuela con la ubicación de Barbacoas y El Tocuyo. En la parte derecha, el mapa del municipio Morán con la posición de Barbacoas y El Tocuyo.

Con la ayuda del Dr. Dieter Korn (curador de la colección de cefalópodos fósiles - MNK) y del geólogo Andreas Abele (responsable de la colección de invertebrados fósiles - MNK) fue posible encontrar un ejemplar dentro de una pequeña colección académica, interpretado dentro de la presente contribución como el utilizado para la descripción e ilustración de Ammonites Leonhardianus por Karsten (1858, p. 106, pl. 2, fig. 5a-b) y propuesto como lectotipo. Este ejemplar se encuentra ahora incluido en la colección principal de invertebrados fósiles junto con otros individuos de la misma especie, que no fueron ilustrados ni descritos por Karsten.

Los ejemplares de esta especie, colectados en Venezuela, son investigados en la presente contribución, por lo 
que se presenta su descripción, fotografías, líneas de sutura, y se dan a conocer los tipos propuestos de la colección Karsten, buscando contribuir a la tipificación de la especie.

\section{Marco Teórico}

Karsten, en sus publicaciones, indica diferentes sitios en relación con las rocas que contienen Ammonites Leonhardianus Karsten, 1858: cerca de "Tucujo" (Karsten, 1858, p. 84, 105); en las montañas de Trujillo (Karsten, 1858, p. 107); en Trujillo (Karsten, 1858, p. 110) o en la provincia de Mérida (Karsten, 1858, p. 111); en la provincia de Trujillo (Karsten, 1886, p. 9); y al norte de "Tucujo" (Karsten, 1886, p. 17). En torno a este tema Sievers (1888, p. 24) hace referencia a "Barbacoas - Schichten, nur bei Barbacoas" (capas de Barbacoas), al igual que Gerhardt (1897a, Schichten von Barbacoas).

Geológicamente, en la zona de Barbacoas, se encuentra una sucesión calcárea del Cretácico en donde aparecen amonitas del Turoniano (cf. Karsten, 1858, 1886, p. 18, 1947, p. 364; Renz, 1982; Klug y Etter, 2013; Patarroyo y Bengtson, 2018) como Ammonites Mosquerae = Benueites mosquerae (Karsten, 1858); Ammonites Toroanus = Vascoceras toroanus (Karsten, 1858); y Ammonites Ospinae = Codazziceras ospinae (Karsten, 1858); y del Coniaciano como Ammonites Leonhardianus $=$ Paralenticeras leonhardianus
(Karsten, 1858), posiblemente Ammonites Barbacoensis = Niceforoceras cf. umbulaziforme (Basse, 1948). También existen algunos depósitos del Cretácico Inferior tal como lo refieren von Buch (1850), Renz (1982), Klug y Etter (2013) con base en Anapuzosia tucuyensis (von Buch, 1850) y Stieler (1920) con base en Venezoliceras karsteni (Stieler, 1920).

La especie Ammonites Leonhardianus Karsten (1858) ha sido referida de forma variada y dentro de diferentes géneros, tal como Pulchellia Leonhardi (Gerhardt, 1897b), Psilotissotia Leonhardi (Roman, 1938), Psilotissotia leonhardiana (Gerth, 1941 en Renz, 1982, p. 10), Psilotissotia Leonhardiana (Gignoux, 1920), Tissotia (Metatissotia) leonhardina (Hubach, 1957), Tissotia (Metatissotia) leonhardiana (Etayo-Serna, 1964), Paralenticeras leonhardianum (Renz, 1982; Kennedy et al., 1995), Hoplitoides leonhardianus ? (Etayo-Serna, 1994) y Discoidellia ? leonhardiana (Vermeulen, 1995, p. 66).

\section{Metodología}

Una búsqueda intensa y cuidadosa dentro de la colección del Museo de Historia Natural de Berlín (MNK), permitió el reconocimiento de los ejemplares fósiles colectados por Hermann Karsten en Venezuela, que están acompañados por etiquetas con datos de la colecta (Figura 2).
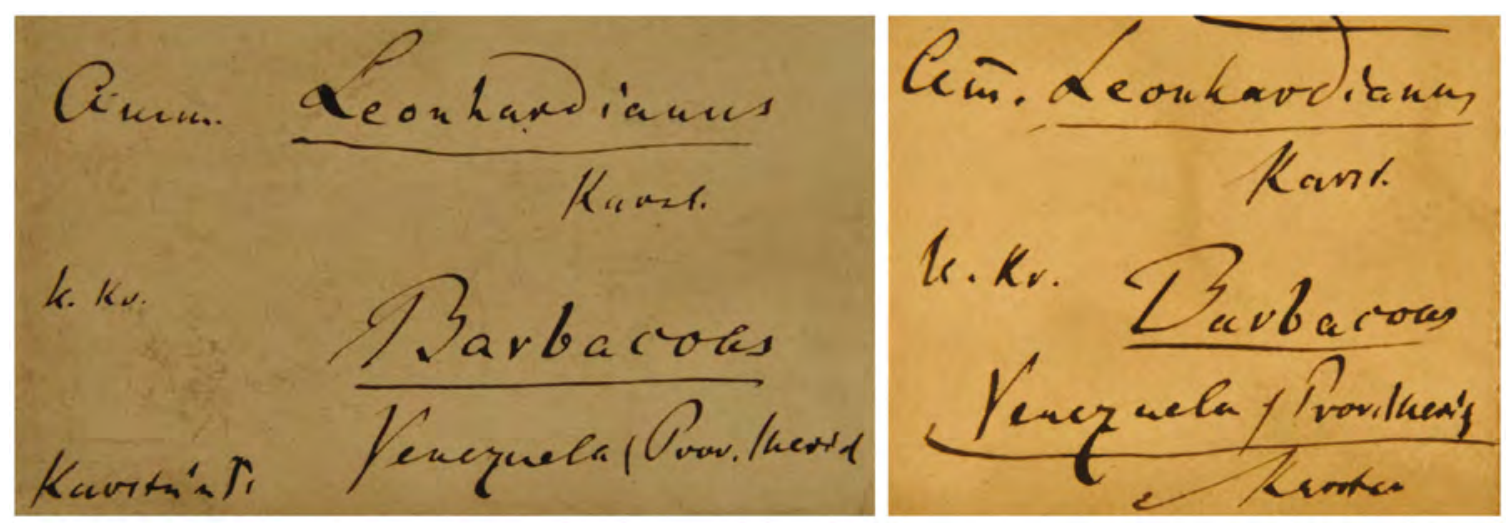

Figura 2. Etiquetas que acompañan a los ejemplares de Paralenticeras leonhardianus (Karsten, 1858).

Las cerca de 30 muestras de Ammonites Leonhardianus Karsten, ya sea individuales o no, están acompañados por el acrónimo MB.C, un número y etiquetas de colecta (Figura 2). Se presentan las medidas obtenidas de 26 individuos asociados a esta especie y que hacen parte de la colección de Karsten proveniente de Barbacoas (Municipio Morán, estado Lara, Venezuela). 
Los individuos de Ammonites Leonhardianus Karsten reportados aquí, incluyen el ejemplar utilizado para la ilustración de Karsten (1858, p. 106, pl. 2, fig. 5) y 25 ejemplares más que aparecen dentro de la colección con etiquetas que indican a Barbacoas (Venezuela) como lugar de colecta (Figura 2).

Para las medidas, sobre ejemplares principalmente completos, se usó un pie de rey, con el que se obtuvo en milímetros el diámetro $=\mathrm{D}$, altura de la vuelta $=$ Wh, ancho de la vuelta $=\mathrm{Ww}$; diámetro umbilical $=\mathrm{U}$. En algunos casos se determinó el número de costillas por vuelta o por media vuelta.

Los fósiles fueron descritos tal cual se encuentran dentro de la colección. Solo el ejemplar propuesto como lectotipo se blanqueó con cloruro de amonio para ser fotografiado, que como se manifestó anteriormente se asume como el que aparece en los dibujos de las láminas de Karsten (1858, pl. 2, fig. 5a-b; 1886; 1947). Las líneas de sutura de algunos ejemplares se dibujaron con una cámara lucida de marca Leica, y posteriormente fueron digitalizadas y editadas.

\section{Resultados}

\section{Taxonomía}

La taxonomía empleada se fundamenta en Wright et al. (1996).

Suborden Ammonitina Hyatt, 1889

Superfamilia Acanthoceratoidea de Grossouvre, 1894

Familia Sphenodicidae Hyatt, 1900

Subfamilia Lenticeratinae Hyatt, 1900

Género Paralenticeras Hyatt, 1900

Especie tipo Amaltheus Sieversi Gerhardt, 1897a, p. 79, fig. 5, pl. I, fig. 5a-b.

\section{Diagnóstico del género}

Con base en las descripciones de Renz (1982, p. 110) y Wright et al. (1996, p. 204) Paralenticeras presenta una concha comprimida con vientre fastigiado, quilla aguda, ombligo profundo y estrecho.

Se debe adicionar que posee sección de la vuelta comprimida y levemente redondeada, con el ancho máximo inmediatamente arriba del margen umbilical. Las costillas principales, secundarias o bifurcadas son sinuosas. Se presenta engrosamiento sobre las costillas cerca del borde umbilical y hacia el sector superior del flanco. Se debe resaltar igualmente, que Wright et al. (1996, p. 204) citan que "Lenticeras and Paralenticeras may be inflated and compressed of the same genus" (Lenticeras y Paralenticeras pueden ser formas infladas o comprimidas del mismo género) y además "suture much as in Lenticeras, but elements deeper and saddles more finely divided" (línea de sutura más como en Lenticeras pero con incisiones más profundas y sillas más finamente divididas).

\section{Denominación específica}

El uso de la denominación específica relacionada con Ammonites Leonhardianus Karsten es variable a lo largo del tiempo, por ejemplo Leonhardianus (Karsten, 1858, 1886, 1947), Leonhardi (Gerhardt, 1897b; Roman, 1938), leonhardiana (Gignoux, 1920; Gerth, 1941 en Renz, 1982; Etayo-Serna, 1964; Vermeulen, 1995), leonhardina (Hubach, 1957), leonhardianum (Renz, 1982; Kennedy et al., 1995), leonhardianus (Etayo-Serna, 1994).

La denominación específica debería ser Paralenticeras leonhardii (Karsten, 1858) con base en las normas establecidas para los nombres propios. Sin embargo, respetando la denominación específica original de Karsten (1858) para el presente trabajo se usa P. leonhardianus.

\section{Tipos}

Con base en las normas de la International Commission on Zoological Nomenclature (ICZN, 1999) se debe proponer un lectotipo y paralectotipos para Ammonites Leonhardianus ya que Karsten (1858) no designó un ejemplar tipo. Muy a pesar que Karsten (1858) no designó claramente el ejemplar utilizado para la ilustración y descripción de A. Leonhardianus (Karsten, 1858, p. 106, pl. 2, fig. 5) indicó que cuenta con un diámetro (D) de 48 $\mathrm{mm}$, altura de la vuelta (Wh) $25 \mathrm{~mm}$, ancho de la vuelta (Ww) 13 mm y diámetro umbilical (U) $5 \mathrm{~mm}$, a partir del cual se hubiese podido proponer un holotipo implícito siguiendo la propuesta de Cunningham et al. (1976). Así que como lectotipo de Ammonites Leonhardianus se propone el individuo MB.C 5781 (Figura 3A-3D), que posee medidas aproximadas a las indicadas por Karsten (1858, p. 106) con diámetro (D) 47,3 mm; altura de la vuelta (Wh) 27,9 mm; ancho de la vuelta (Ww) 13,3 ? mm y diámetro umbilical (U) 6,8 ? mm. Adicionalmente, se trata del individuo más grande de Paralenticeras leonhardianus de la colección Karsten. 


\section{Material}

Aproximadamente 30 individuos provenientes de Venezuela, colectados por Hermann Karsten, se encuentran dentro de la colección de invertebrados fósiles del Museo de Historia de Berlín (MNK). Los datos obtenidos de 26 ejemplares se citan en la Tabla 1.
También es posible proponer como paralectotipos los ejemplares MB.C29209 (Figura 4J-4K; en parte molde interno), MB.C29210 (Figura 3H-3I; molde interno del fragmócono) y MB.C29230 (Figura 6I-6J; molde interno).

Paralenticeras leonhardianus (Karsten, 1858)

Figura 3 (A-O), Figura 4 (A-K), Figura 5 (A-W), Figura 6 (A-L), Figuras 7-12.

v* 1858 Ammonites Leonhardianus n. sp. Karsten, p. 106, pl. 2, fig. 5a-b.

v * $1886 \quad$ Ammonites Leonhardianus Karsten, Karsten, pl. 2, fig. 5a-b.

non 1897b Pulchellia Leonhardi Karsten, Gerhardt, p. 155, pl. 3, fig. 10.

1920 Psilotissotia leonhardiana (Karsten), Gignoux, p. 137.

1938 Psilotissotia Leonhardi Karsten, Roman, p. 471.

v * $1947 \quad$ Ammonites Leonhardianus Karsten, p. 374, pl. 2, cuarta fila dos figuras a la izquierda.

? 1957 Tissotia (Metatissotia) leonhardina, Hubach, p. 43.

1964 Tissotia (Metatissotia) leonhardiana (Karsten), Etayo-Serna, p. 125.

1982 Paralenticeras leonhardianum (Karsten), Renz, p. 110, fig. 84, pl. 36, figs. 2-6.

non $1994 \quad H o p l i t o i d e s ~ l e o n h a r d i a n u s$ (Karsten) ?, Etayo-Serna, p. 6, fig. 2a, b, pl. 1, fig. 5.

1995 Discoidellia ? leonhardiana (Karsten), Vermeulen, p. 66.

1995 Paralenticeras leonhardianum (Karsten), Kennedy et al., p. 425.

Tabla 1. Medidas en mm y relaciones de medida de 26 ejemplares de Paralenticeras leonhardianus (Karsten, 1858).

\begin{tabular}{|c|c|c|c|c|c|c|c|}
\hline Muestra \# & D & Wh & $\mathbf{W w}$ & $\mathbf{U}$ & Wh/D & Ww/Wh & U/D \\
\hline \multirow[t]{2}{*}{ MB.C5781 } & 47,3 & 27,9 & $13,3 ?$ & $6,8 ?$ & 0,590 & $0,477 ?$ & 0,144 ? \\
\hline & 35 & 18,7 & 11,3 & 6,2 & 0,534 & 0,604 & 0,177 \\
\hline \multirow[t]{2}{*}{ MB.C29221 } & 21,2 & 10,3 & 6,3 & 3,9 & 0,486 & 0,612 & 0,184 \\
\hline & 19,8 & 9,4 & 6,4 & $3,4 ?$ & 0,475 & 0,681 & 0,172 ? \\
\hline MB.C29230 & 21,6 & 10 & 6,7 & 4,3 & 0,463 & 0,670 & 0,199 \\
\hline MB.C29231 & 21 & 11 & 5,9 & 3,3 & 0,524 & 0,536 & 0,157 \\
\hline MB.C29232 & 19,3 & 10,2 & 5,9 & 3 & 0,528 & 0,578 & 0,155 \\
\hline MB.C29239 & 21,6 & 11,2 & 7,4 & $3,4 ?$ & 0,519 & 0,661 & $0,157 ?$ \\
\hline MB.C29238 & 14 & 6,5 & & $3,2 ?$ & 0,464 & & $0,229 ?$ \\
\hline MB.C29237 & 24,4 & 13,4 & 7,1 & $4 ?$ & 0,549 & 0,530 & $0,164 ?$ \\
\hline MB:C29236 & 22,5 & 12,4 & $6,9 ?$ & $3,2 ?$ & 0,551 & $0,556 ?$ & $0,142 ?$ \\
\hline MB.C 29240 & & 10,9 & 7,1 & & & 0,651 & \\
\hline MB.C 29241 & & 15,9 & 9,1 & & & 0,572 & \\
\hline MB.C29209 & 34,7 & 18,1 & 9,7 & $5,8 ?$ & 0,522 & 0,536 & 0,167 ? \\
\hline MB.C29210 & 23,7 & 11,9 & 7,3 & 4,3 & 0,502 & 0,613 & 0,181 \\
\hline MB.C29208 & & 14,8 & 13 & 4,4 & & 0,878 & \\
\hline MB.C29213 & 35,8 & 18,2 & 10,2 & 5,7 & 0,508 & 0,560 & 0,159 \\
\hline MB.C29206 & & 21 & 10,6 & $5,9 ?$ & & 0,505 & \\
\hline MB.C29220 & 25,5 & 14,8 & 8,2 & 3 & 0,580 & 0,554 & 0,118 \\
\hline MB.C29224 & 17 & 9 & 5 & 3,1 & 0,529 & 0,556 & 0,182 \\
\hline MB.C29222 & 17,3 & 9,3 & 5,4 & 2,7 & & 0,581 & \\
\hline MB.C29234 & 17,5 & $9 ?$ & 4,9 & & & $0,544 ?$ & \\
\hline MB.C29228 & 14,8 & $8 ?$ & 4,8 & & 0,541 & 0,600 & \\
\hline MB.C 29205 & 35 & 17,3 & & $5,6 ?$ & 0,494 & & $0,160 ?$ \\
\hline MB.C29227 & 17,2 & $8,4 ?$ & 5,5 & & & $0,655 ?$ & \\
\hline MB.C29226 & & 11,2 & 6,4 & & & 0,571 & \\
\hline MB.C29229 & & 7,9 & 4,5 & & & 0,570 & \\
\hline MB.C29225 & & 13,6 & 7,9 & & & 0,581 & \\
\hline
\end{tabular}



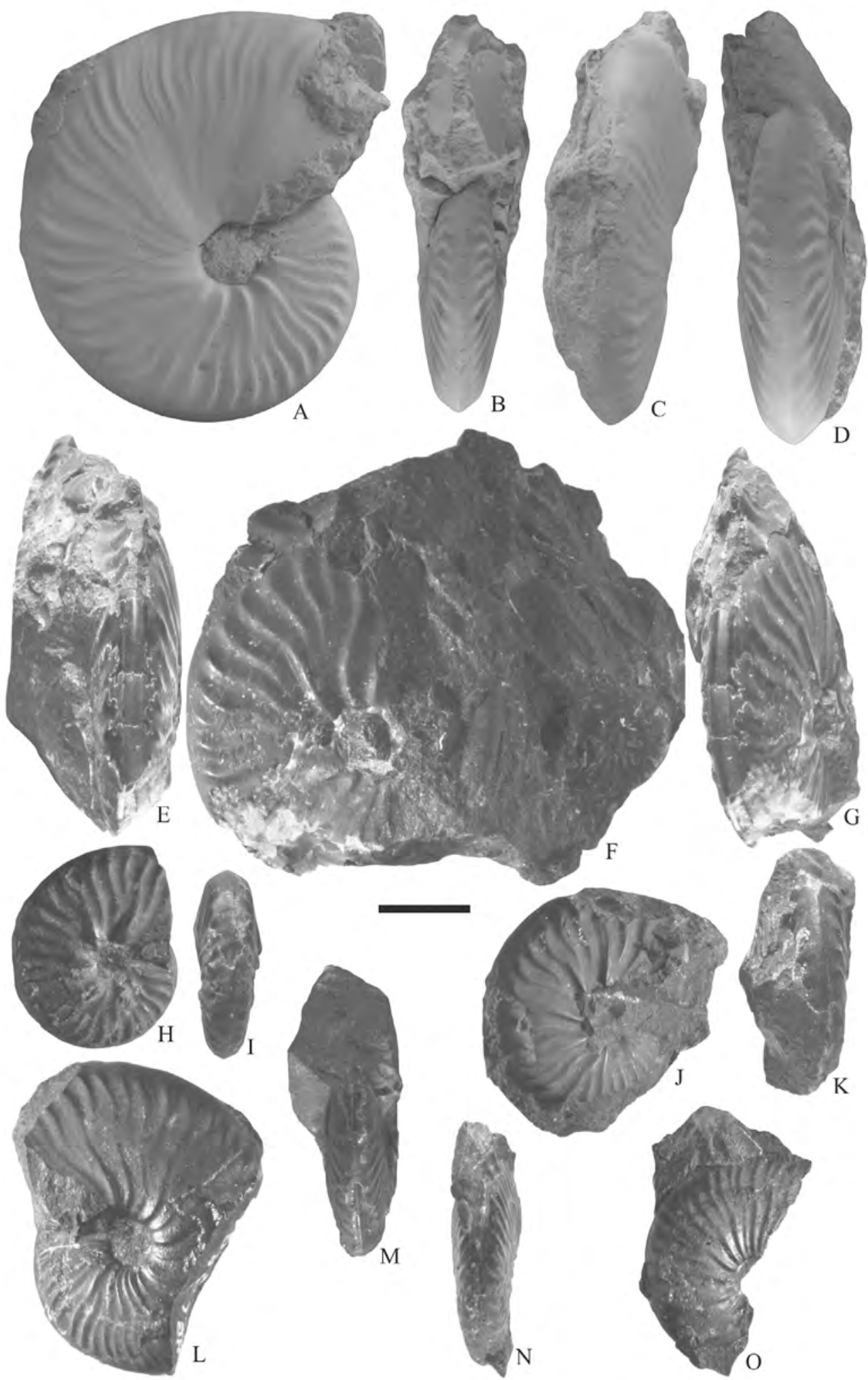

Figura 3. Paralenticeras leonhardianus (Karsten, 1858), del Coniaciano de Barbacoas, Venezuela. A-D. Lectotipo MB.C5781, vistas lateral (A), oral (B), aboral (C), oral inferior (D). E-G. MB.C29206, vistas aboral (E), lateral (F), aboral y lateral (G). H-I. Paralectotipo MB.C29210, vistas lateral (H), aboral (I). J-K. MB.C29212, vistas lateral (J), aboral (K). L-M. MB.C29213, vistas lateral (L), oral (M). N-O. MB.C29220, vistas aboral (N), lateral (O). Escala gráfica $10 \mathrm{~mm}$. 


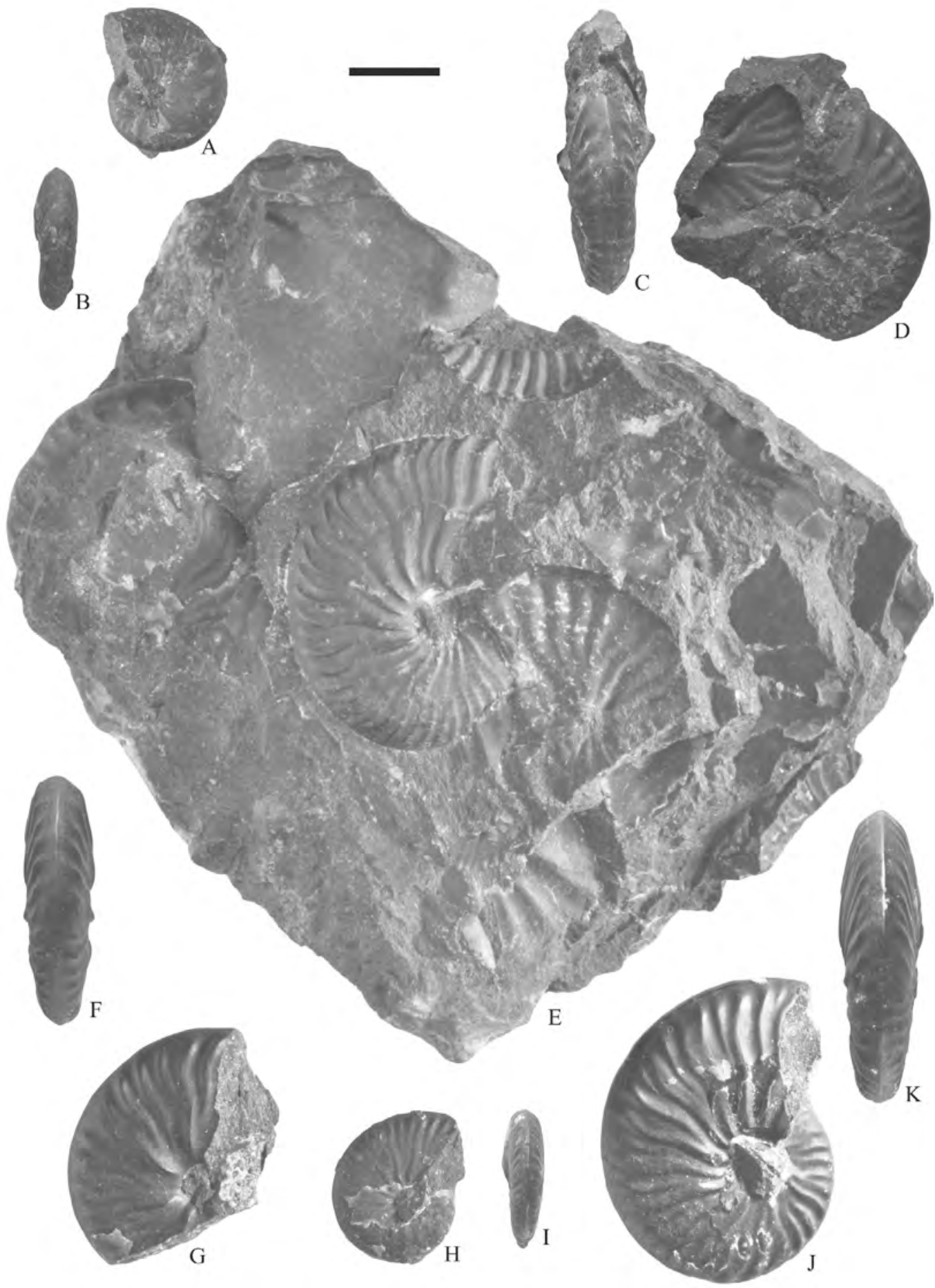

Figura 4. Paralenticeras leonhardianus (Karsten, 1858), del Coniaciano de Barbacoas, Venezuela. A-B. ejemplar MB.C29222 vistas lateral (A), aboral (B). C-D. MB.C29207 vistas aboral (C), lateral (D). E. MB.C29205 vistas lateral. F-G. MB.C29208 vistas aboral (F), lateral (G). H-I. MB.C29224 vistas lateral (H), aboral (I). J-K. Paralectotipo MB.C29209 vistas lateral (J), aboral (K). Escala gráfica $10 \mathrm{~mm}$. 

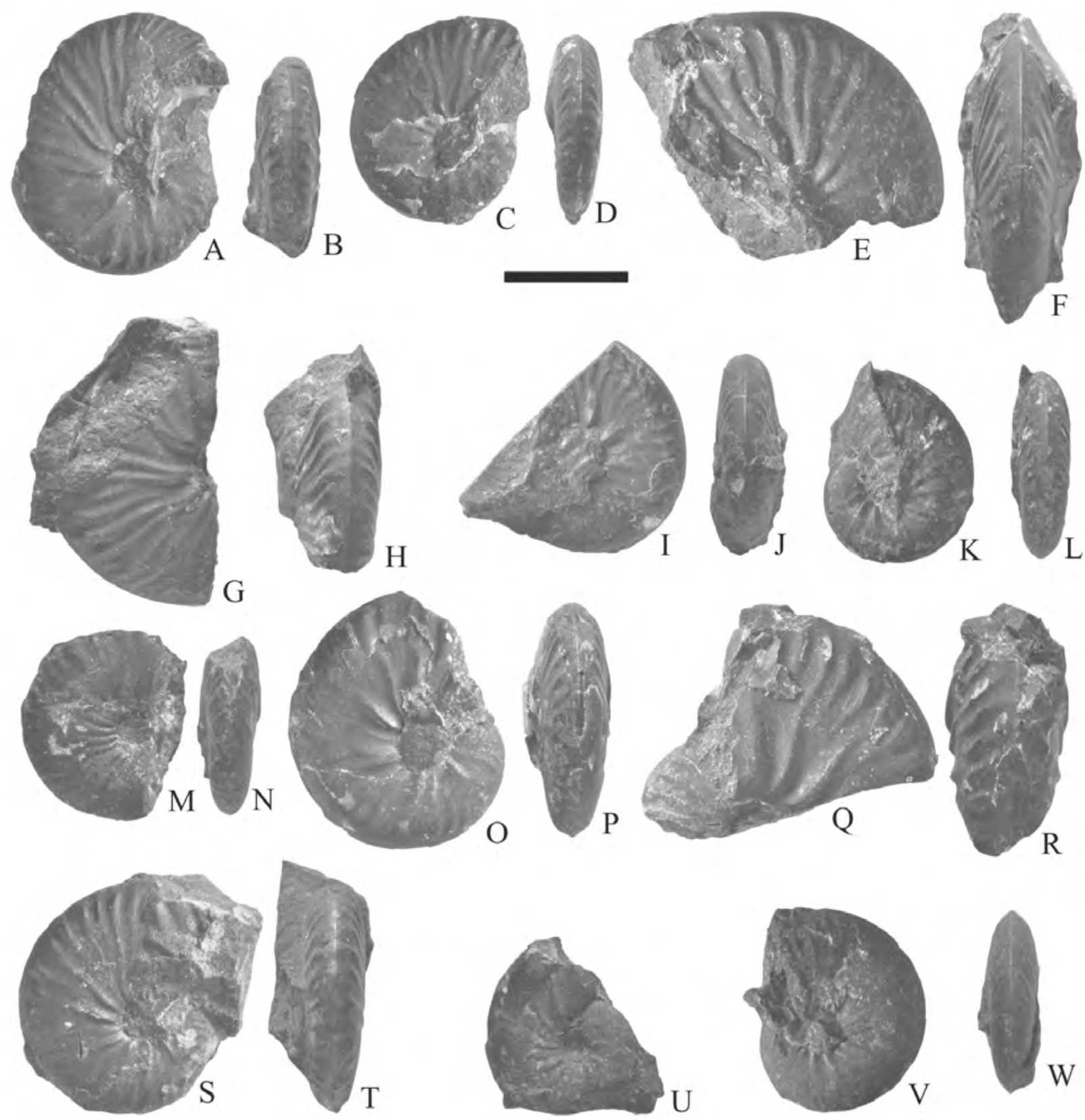

Figura 5. Paralenticeras leonhardianus (Karsten, 1858), del Coniaciano de Barbacoas, Venezuela. A-B. ejemplar MB.C29221 vistas lateral (A), aboral (B). C-D. MB.C29224 vistas lateral (C), aboral (D). E-F. MB.C29225 vistas lateral (E), aboral (F). G-H. MB.C29226 vistas lateral (G), aboral (H). I-J. MB.C29227 vistas lateral (I), aboral (J). K-L. MB.C29228 vistas lateral (K), aboral (L). M-N. MB.C29229 vistas lateral (M), aboral (N). O-P. MB.C29239 vistas lateral (O), aboral (P). Q-R. MB.C29241 vistas lateral (Q), aboral (R). S-T. MB.C29232 vistas lateral (S), aboral (T). U. MB.C29233 vistas lateral. V-W. MB.C29234 vistas lateral (V), aboral (W). Escala gráfica $10 \mathrm{~mm}$. 

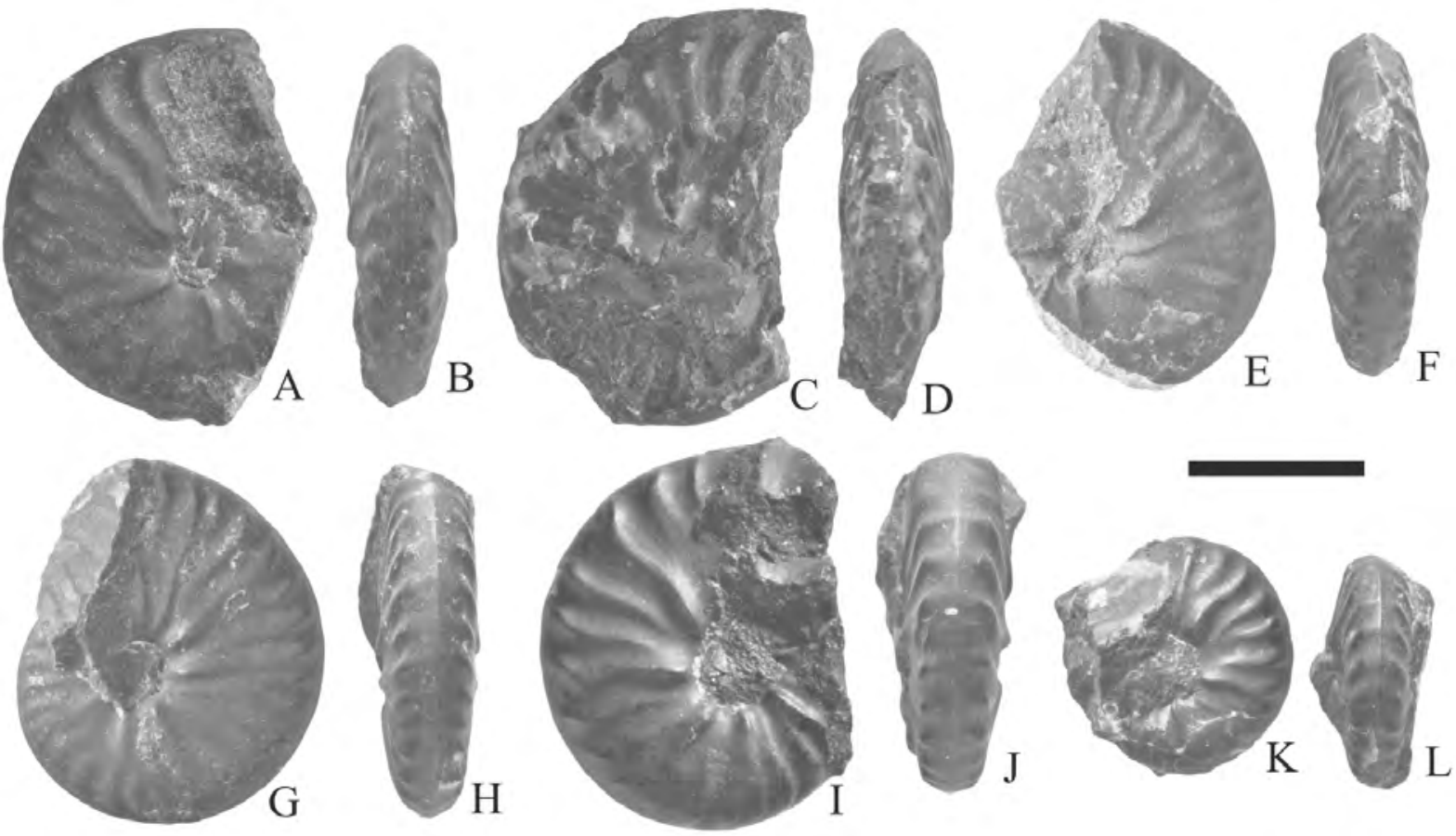

Figura 6. Paralenticeras leonhardianus (Karsten, 1858), del Coniaciano de Barbacoas, Venezuela. A-B. MB.C29236 vistas lateral (A), aboral (B). C-D. MB.C29237 vistas lateral (C), aboral (D). E-F. MB.C29240 vistas lateral (E), aboral (F). G-H. MB.C29231 vistas lateral (G), aboral (H). I-J. Paralectotipo MB.C29230 vistas lateral (I), aboral (J). K-L. MB.C29238 vistas lateral $(\mathrm{K})$, aboral (L). Escala gráfica $10 \mathrm{~mm}$.

\section{Repositorio}

Colección Karsten dentro de la colección de invertebrados fósiles del Museo de Historia Natural de Berlín (Museum für Naturkunde - MNK), Alemania.

\section{Descripción}

El lectotipo (MB.C5781) es un molde interno con fragmócono y cámara de habitación, preservado en material calcáreo negro (Figura 3A-3D). La sección de la vuelta es lanceolada, extremadamente discoidal, subinvoluta (0,177\% U/D) siguiendo a Korn (2010). La cámara de habitación preservada ocupa algo más de la mitad de la vuelta. Las costillas primarias son sinuosas (falcoides) con fuerte proyección hacia el peristoma en la parte alta del flanco y terminan al final de la zona ventrolateral cerca de la quilla sifonal. Entre las costillas primarias se presentan costillas secundarias largas, intermedias o cortas, que siempre se van relevando desde la más larga hasta la más corta cuando aparecen dos o tres. Algunas de estas inician en el flanco medio inferior, medio o medio superior. Las costillas son en general intermedias y espaciadas, pero al final de la última vuelta se presentan más finas y un poco menos espaciadas. Las costillas principales muestran bullas cerca del borde umbilical, mientras que todas las costillas poseen tubérculos ventrolaterales, que en estadio adulto no son destacados. Desde los $44 \mathrm{~mm}$ de altura de la vuelta las costillas son más numerosas, menos pronunciadas y hacia el flanco inferior tienen la apariencia de líneas de crecimiento. La quilla es menos evidente en estado adulto, sobre la que las líneas de crecimiento producen una leve crenulación. La bifurcación se presenta sobre algunas costillas. El máximo espesor del ancho de la vuelta se da en la parte más baja del flanco y cerca del borde umbilical. 46 costillas medias y finas se reconocen en la última vuelta.

Entre 30 (MB.C29210) y 36 (MB.C29209) costillas están presentes sobre la última vuelta en individuos que no muestran costillas finas (Figura 3H-3I, Figura 4J-4K). MB.C29230 y MB.C29238 presentan sección de la vuelta subrectangular a lanceolada (Figuras 6I-6J, $6 \mathrm{~K}-6 \mathrm{~L}$ ), hombros ventrolaterales, costillas algo gruesas y suavemente curvadas hacia el margen ventrolateral. Las costillas secundarias nacen hacia el flanco medio inferior, medio y medio superior. 11 (MB.C29238) o 13 (MB.C29230) costillas espaciadas pueden estar en media vuelta. La quilla en estos ejemplares es tenue. Fuertes tubérculos se desarrollan cerca del borde umbilical. 
En estadio joven, la concha es poco ornamentada y lisa, cerca de 3,7 mm (MB.C29233, MB.C29234), $4 \mathrm{~mm}$ (MB.C29228) o 4,6 mm (MB.C29222) de altura de la vuelta aparecen costillas suaves y luego tubérculos periumbilicales (Figuras 4A-4B, 5K-5L, 5U-5W). Además, la quilla ya está presente.

En MB.C29222 la cámara de habitación ocupa mínimo tres cuartas partes de la espira (Figura 4A-4B).

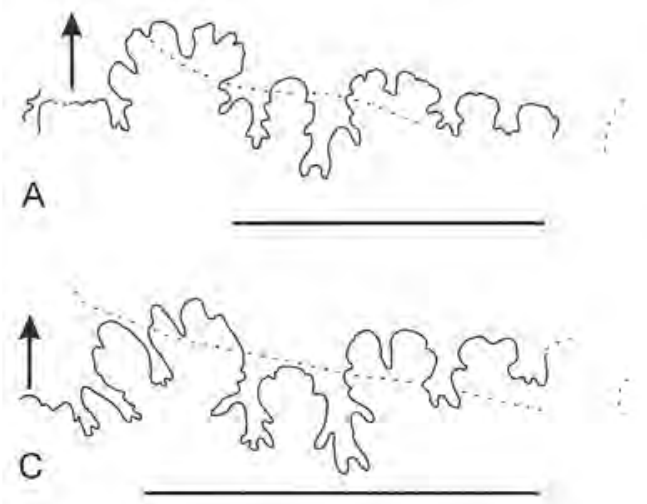

MB.C29206 (Figura 3E-3G) y MB.C29225 (Figura $5 \mathrm{E}-5 \mathrm{~F}$ ) presentan el área ventral más aguda con una faceta sobre una concha muy discoidal.

MB.C29220 (Figura 3N-3O), MB.C29213 (Figura 3L3M), y MB.C29229 (Figura 5M-5N) muestran costillas finas y escasa tuberculación.

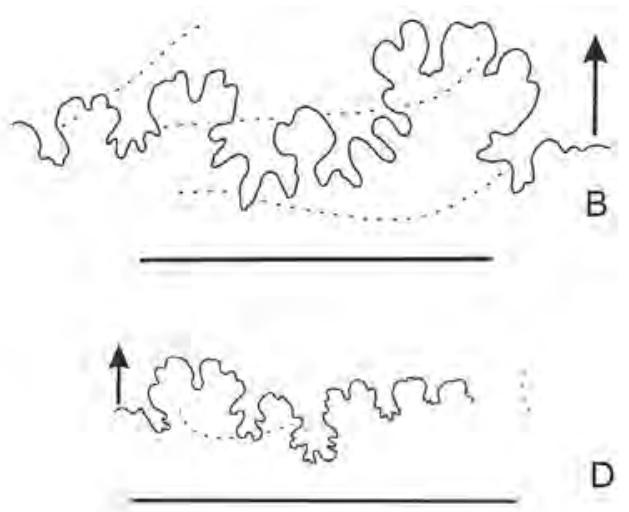

Figura 7. Líneas de sutura de Paralenticeras leonhardianus (Karsten, 1858). A. MB.C5781, lectotipo. B. MB.C29206. C. MB.C29209, paralectotipo. D. MB.C29236. Escala gráfica $10 \mathrm{~mm}$.

La línea de sutura presenta la silla lateral amplia, redondeada y con incisiones relativamente profundas, y los lóbulos igualmente muestran incisiones profundas (Figura 7).

El crecimiento de la concha es variable, lo que se hace evidente comparativamente con las relaciones $\mathrm{D}$ y $\mathrm{U}$ (Figura 8), U y Ww (Figura 9), pero progresiva teniendo en cuenta las relaciones D y Wh (Figura 10), D y Ww (Figura 11). La relación U/D muestra el comportamiento normal de una población con base en un análisis estadístico convencional (Figura 12), lo que significa que hacia los extremos aparecen las formas menos frecuentes mientras que la tendencia central agrupa las formas más abundantes.

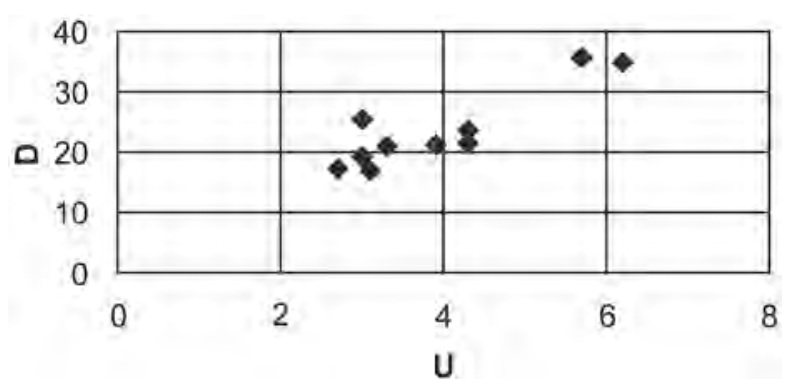

Figura 8. Gráfico de la relación de D (diámetro) y U (diámetro umbilical) en mm de Paralenticeras leonhardianus (Karsten, 1858).
Figura 9. Gráfico de la relación de U (diámetro umbilical) y Ww (ancho de la vuelta) en $\mathrm{mm}$ de Paralenticeras leonhardianus (Karsten, 1858).

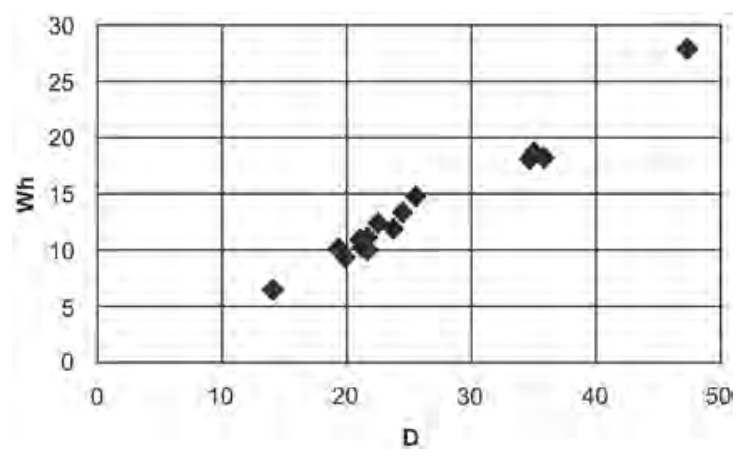

Figura 10. Gráfico de la relación de D (diámetro) and Wh (alto de la vuelta) en mm de Paralenticeras leonhardianus (Karsten, 1858). 


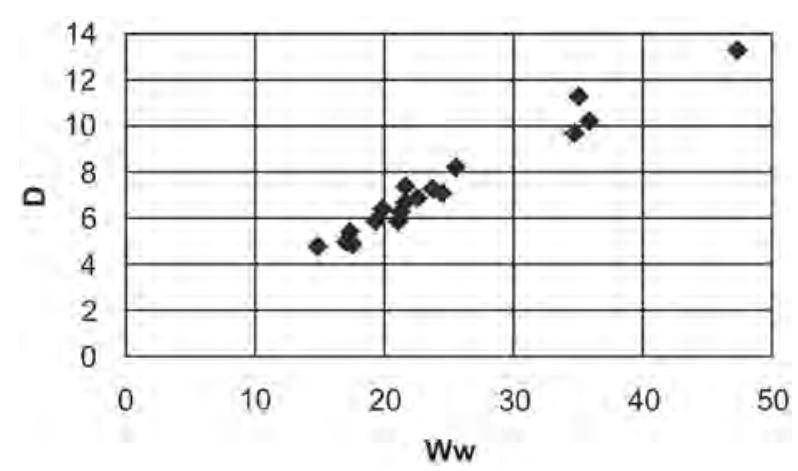

Figura 11. Gráfico de la relación de D (diámetro) and $\mathrm{Ww}$ (ancho de la vuelta) en mm de Paralenticeras leonhardianus (Karsten, 1858).

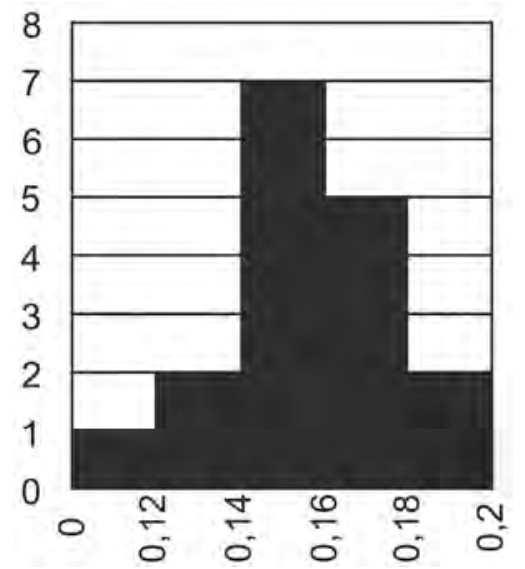

Figura 12. Histograma de la relación U/D de Paralenticeras leonhardianus (Karsten, 1858).

\section{Discusión}

La variabilidad en la población de Paralenticeras leonhardianus (Karsten, 1858) se hace evidente en diferentes aspectos. Formas poco frecuentes aparecen con costillas finas y tuberculación suave MB.C29220 (Figura 3N-3O), MB.C29213 (Figura 3L-3M) y MB.C29229 (Figura 5M-5N); mientras que otras poseen costillas gruesas, espaciadas y tubérculos más pronunciados MB.C29230 y MB.C29238. Igualmente MB.C29206 (Figura 3E-3G) y MB.C29225 (Figura 5E-5F) representan individuos con el área ventral más aguda y con una faceta sobre una concha muy discoidal, mientras que al otro extremo MB.C29230 y MB.C29238 muestran sección de la vuelta subrectangular (Figuras 6I-6J, 6K-6L) con hombros ventrolaterales.

Si bien MB.C 29206 (Figura 3E-3G) y MB.C29225 (Figura 5E-5F) muestran conchas lanceoladas con una faceta ventral como en algunas especies de
Eulophoceras (cf. E. austriacum en Kennedy et al., 1995, pl. 25, fig. 3-5; E. jacobi en Patarroyo y Dueñas, 2006, pl. I, fig. 1-3; Patarroyo, 2011, pl. 4, fig. 7-10; Kennedy y Klinger, 2012), desde luego no implica que deban ser asociados con dicho género, más aún cuando la costulación y tuberculación es la típica de $P$. leonhardianus (Karsten, 1858).

P. leonhardianus (Karsten) es la especie más ornamentada del género Paralenticeras mientras que P. sieversi (Gerhardt, 1897b), P. spathi Reyment, 1958 y P. caneroti Collignon, 1983 (Kennedy et al. 1995, pl. 25, fig. 8-10) muestran pobre ornamentación, más pliegues y líneas de crecimiento.

\section{Distribución}

Paralenticeras leonhardianus (Karsten, 1858), hasta ahora, está reportada únicamente en Venezuela, aunque se encuentra citada sin ilustración para Colombia como Tissotia (Metatissotia) leonhardina (cf. Hubach, 1957, p. 43).

\section{Rango estratigráfico}

Para el género Paralenticeras Hyatt, 1900, Wright et al. (1996, p. 204) establecen un rango estratigráfico entre el Coniaciano superior y el Santoniano. Renz (1982, p. 110, fig. 91) reporta para Paralenticeras leonhardianus (Karsten, 1858) un rango del Coniaciano inferior teniendo en cuenta su hallazgo en la sucesión inferior del Miembro Timbetes de la Formación "La Luna" en la sección cerro Gordo a $4 \mathrm{~km}$ al NE de Barbacoas (Venezuela).

Así que, se hace necesario lograr una descripción detallada de la sucesión del Cretácico Superior en el sector de Barbacoas (Venezuela), con el fin de establecer una bioestratigrafía fiable. Además, existe la posibilidad que $P$. leonhardianus haya sido colectada junto con Ammonites barbacoensis (=Niceforoceras cf. umbulaziforme Basse, 1948), cuyo rango estratigráfico es del Coniaciano.

\section{Conclusiones}

El Ammonites Leonhardianus (Karsten, 1858, pl. 6, fig. 6) de Barbacoas (municipio Morán, estado Lara Venezuela) etiquetado como MB.C5781 es propuesto como lectotipo de Paralenticeras leonhardianus Karsten (1858). 
26 ejemplares de Venezuela, de la colección de Karsten, fueron investigados, descritos y fotografiados con el fin de reforzar la tipificación de Paralenticeras leonhardianus. De estos se proponen como paralectotipos los ejemplares MB.C29209, MB.C29210 y MB.C29230.

Se acepta un rango estratigráfico de Coniaciano para Paralenticeras leonhardianus (Karsten, 1858) sin poderse restringir al Coniaciano inferior como lo indica Renz (1982), ya que en la presente investigación no se cuenta con amarre estratigráfico.

\section{Agradecimientos}

Ésta contribución es una parte de mi visita de investigación al Museo de Historia Natural de Berlín entre abril y junio de 2019 bajo el auspicio del DAAD beca 2019 (57440916). Deseo manifestar mi agradecimiento al Prof. Dr. Kai Wünnemann, a los doctores Dieter Korn, Oliver Hampe, Christian Neumann y al geólogo Andreas Avele por todo el apoyo prestado durante mi visita de investigación para tener acceso a la colección Karsten. Igualmente, agradezco de forma especial a los evaluadores doctores Fernando Barroso-Barcenilla (Dpto. de Geología, Geografía y Medio Ambiente, Universidad de Alcalá, España) y Josep A. Moreno-Bedmar (Instituto de Geología, Universidad Nacional Autónoma de México, México) por sus valiosos comentarios que contribuyeron al mejoramiento de la presente contribución.

\section{Referencias}

Aalto, K.R. (2015). Hermann Karsten, pioneer of geologic mapping in northwestern South America. History of Geo- and Space Sciences, 6(1), 57-63. https://doi.org/10.5194/hgss-6-57-2015

Basse, E. (1948). Quelques ammonites nouvelles du Crétacé de Colombie (Am. Sud). Bulletin de la Société Géologique de France, S5-XVIII(8-9), 691-698. https://doi.org/10.2113/gssgfbull.S5XVIII.8-9.691

Carrillo-Briceño, J.D.; Amson, E.; Zurita, A.; SánchezVillagra, M.R. (2016). Hermann Karsten (18171908): a German naturalist in the Neotropics and the significance of his paleovertebrate collection. Fossil Record, 20, 21-36. https://doi.org/10.5194/ fr-20-21-2016
Cunningham, J.L.; Bakshi, B.K.; Lentz, P.L.; Gilliam, M.S. (1976). Two new genera of leaf-parasitic fungi (Basidiomycetidae: Brachybasidiaceae). Mycologia, 68(3), 640-654. https://doi. org/10.2307/3758985

de Grossouvre, A. (1894). Recherches sur la craie supérieure, 2, Paléontologie. Les ammonites de la craie supérieure. Mémoires pour servir à l'explication de la Carte Géologique détaillée de la France (pp. 1-264). Imprimerie nationale.

Etayo-Serna, F. (1964). Posición de las Faunas en los depósitos cretácicos colombianos y su valor en la subdivisión cronológica de los mismos. Boletín de Geología, 16-17, 5-142.

Etayo-Serna, F. (1994). Age of the mosasaur-bearing beds, Upper Magdalena Valley, Colombia. En: F. Etayo-Serna (ed.). Estudios Geológicos del Valle Superior del Magdalena (pp. XVI 1 - XVI 9). W. Taller Editorial Ltda.

Gerhardt, K. (1897a). Beitrag zur kenntniss der Kreideformation in Venezuela und Peru. En: G. Steinmann (ed.). V. Beiträge zur Geologie und Paläontologie von Südamerika. Neues Jahrbuch für Mineralogie, Geologie und Paläontologie, Beilage-Band, XI, 65-117.

Gerhardt, K. (1897b). Beitrag zur kenntniss der Kreideformation in Columbien. En: G. Steinmann (ed.). VI. Beiträge zur Geologie und Paläontologie von Südamerika, Neues Jahrbuch für Mineralogie, Geologie und Paläontologie, Beilage-Band, XI, 118-208.

Gignoux, M. (1920). Les Pulchelliidés du Paléocrétacé. En: Mémoire de la Carte Géologique de la France (pp. 135-164). Imprimerie nationale.

González, L.; Castrillo, J.; Hervoüet, Y.; Dhont, D.; Odreman, O.; Bongiorno, F.; Mazuera, F. (2018). Tectónica transpresiva e inversión de Grabenes Jurásicos en Los Andes Septentrionales Venezolanos, región de Barbacoas-Los Humocaros estado Lara y Árbol Redondo-Santa Ana estado Trujillo, Venezuela. Ciencia e Ingeniería, 39(2), 137-146.

Hubach, E. (1957). Contribución a las unidades estratigráficas de Colombia. Instituto Geológico Nacional - Colombia, Informe 1212, 166 pp. 
Hyatt, A. (1889). Genesis of the Arietitidae. Smithsonian Contributions to Knowledge, 26(673), 1-238.

Hyatt, A. (1900). Cephalopoda. En: K.A. von Zittel, 1896-1900, Textbook of Palaeontology (transl. Eastman, C.R.) (pp. 502-604). Macmillan.

ICZN (1999). International Code of Zoological Nomenclature. Padova, Italia. Tipografia La Garangola.

Karsten, H. (1849). Über die Geologie von Venezuela, Bericht über die zur Bekanntmachung geeigneten Verhandlungen der Königlich Preussischen Akademie der Wissenschaften zu Berlin, (im Monat Juli 1849), 197-200.

Karsten, H. (1850). Beitrag zur Kenntniss der Gesteine des nördlichen Venezuela. Zeitschrift der Deutschen Geologischen Gesellschaft, 2, 345361.

Karsten, H. (1858). Über die geognostischen Verhältnisse des westlischen Columbien, der heutigen Republiken Neu-Granada und Equador. Amtlische Bericht über die 32 Versammlungen der Deutscher Naturforscher und Ärzte zu Wien 1856, 80-115.

Karsten, H. (1886). Géologie de l'ancienne Colombie bolivarienne, Venezuela, Nouvelle-Grenade et Ecuador. R. Friedländer \& Sohn.

Karsten, H. (1947). La situación geognóstica de la Nueva Granada. Conferencia dictada en Viena - 1856. Revista de la Academia Colombiana de Ciencias Exactas, Física y Naturales, 7(27), 361380.

Kennedy, W.J.; Bilotte, M.; Melchior, P. (1995). Ammonite faunas, biostratigraphy and sequence stratigraphy of the Coniacian-Santonian of the Corbières (NE Pyrénées). Bulletin des centres de recherches Exploration-Production ElfAquitaine, 19(2), 377-499.

Kennedy, W.J.; Klinger, H.C. (2012). Cretaceous faunas from Zululand and Natal, South Africa. The Santonian-Campanian ammonite genus Eulophoceras Hyatt, 1903. African Natural History, 8, 30-54.
Klug, C.; Etter, W. (2013). Wenig bekannte Fossilien: Cephalopoden aus Venezuela. Fossilien, 30(1), 29-34.

Korn, D. (2010). A key for the description of Palaeozoic ammonoids. Fossil Record, 13(1), 5-12. https:// doi.org/10.1002/mmng.200900008

Patarroyo, P. (2011). Sucesión de amonitas del Cretácico Superior (Cenomaniano - Coniaciano) de la parte más alta de la Formación Hondita y de la Formación Loma Gorda en la quebrada Bambucá, Aipe - Huila (Colombia, S. A.). Boletín de Geología, 33(1), 69-92.

Patarroyo, P.; Dueñas, H. (2006). Eulophoceras jacobi Hourcq y palinomorfos asociados del Coniaciano temprano, Formación Loma Gorda, Quebrada Bambucá (Aipe-Huila-Colombia, S.A.). Revista de la Academia Colombiana de Ciencias Exactas, Físicas y Naturales, 30(117), 503-510.

Patarroyo, P.; Bengtson, P. (2018). Codazziceras ospinae (Karsten, 1858) from the Turonian (Upper Cretaceous) of Colombia. Cretaceous Research, 88, 392-398. https://doi.org/10.1016/j. cretres.2017.04.002

Renz, O. (1982). Cretaceous Ammonites of Venezuela. Basilea, Suiza. Birkhäuser Ed.

Reyment, R. (1958). Uber einige Ammoniten aus dem Coniac Kolumbiens und Venezuelas, Sudamerika. Stockholm Contributions in Geology, 2(1), 1-25.

Roman, F. (1938). Les ammonites Jurassiques et Crétacées. Masson et Cie. Ed.

Sievers, W. (1888). Die Cordillere von Mérida nebst Bemerkungen über das karibische Gebirge. Geographische Abhandlungen, 3(1), 1-338.

Stieler, C. (1920). Über sogennante Mortoniceraten des Gault. Centralblatt für Mineralogie, Geologie und Pläontologie, 345-352, 392-400.

Vermeulen, J. (1995). Nouvelle tripartition de la famille des Pulchelliidae (Ammonoidea) illustree par la descripcion de trois espéces des Alpes de Haute Provence. Riviéra Scientifique, 12, 65-80. 
von Buch, L. (1850). Die Anden von Venezuela.

Zeitschrift der Deutschen Geologischen Gesellschaft, 2, 339-345.

Wright, C.W.; Callomon, J.; Howarth, J. (1996). Cretaceous Ammonoidea. En: R.L. Kaesler (ed.). Treatise on Invertebrate Paleontology (pp. 1-362). Part L. Mollusca 4 Revised, Geological Society of America \& University of Kansas.

Fecha de recibido: 15 de agosto de 2020

Fecha de aprobado: 25 de febrero de 2021 